\title{
Cuidados de enfermería en las etapas del proceso donación-trasplante en chile, aplicado a un caso clínico
}

\author{
*Pía Andrea Hermosilla González, ** Susana Rocha Ortiz, *** Magali Rodríguez Vidal
}

* Enfermera, Magíster en Enfermería, Especialista en Diálisis y Trasplante Renal, Coordinadora Local de

Procuramiento, Unidad de Procuramiento y Trasplante, Hospital Dr. Guillermo Grant Benavente

** Enfermera Unidad de Procuramiento y Trasplante, Hospital Dr. Guillermo Grant Benavente

*** Profesor Asistente, Magíster en Enfermería, Especialista en Diálisis y Trasplante Renal

Universidad Concepción

\section{Introducción}

El trasplante es el tratamiento de elección de la insuficiencia renal crónica terminal, el cual permite una mayor sobreviva y mejor calidad de vida para los usuarios, y un menor costo para el sistema sanitario. Corresponde al resultado de un proceso múltiple y complejo, que requiere organización independiente con recursos adicionales y su éxito depende de la sociedad, de un trabajo en equipo multidisciplinario y la donación de órganos ${ }^{(1)}$.

El procuramiento (obtención) de órganos ha sido definido como todo el proceso mediante el cual se logra que los órganos y tejidos de alguien que ha fallecido, sean implantados en un receptor determinado ${ }^{(2)}$, siendo principalmente el profesional de enfermería el encargado de coordinar todas las actividades derivadas de los diferentes procesos ${ }^{(3)}$, desde la pesquisa del donante hasta la implantación del órgano(4). Su capacitación continua resulta necesaria para llevar a cabo esta práctica(5).

En el mundo, la evolución del procuramiento de órganos, está relacionado con el desarrollo de diversos factores,

Correspondencia:

Pía Andrea Hermosilla González

San Martin número 1436,

Concepción - Chile

E-mail:phermosilla@udec.cl tales como el desarrollo de programas estatales de donación cadavérica, que garanticen la obtención de órganos para trasplante de forma regular; una visión global de lo que representa el trasplante de órganos; el desarrollo de programas paralelos y/o complementarios de donación en vivo; la falta de recursos económicos en países con pocos desarrollo en donde el trasplante no es una prioridad, el soporte gubernamental total y absoluto desde el punto de vista social-político-sanitario y, paralelamente, el desarrollo cultural de la población; y el desarrollo de leyes de trasplante, autorización de centros y registros de regulación de la actividad. Por tanto, el cómo funciona la ayuda del gobierno, la cultura, la religión y la legislación son la clave del resultado para la organización de la generación y trasplante de órganos $^{(6)}$.

Chile en los últimos años, debido a la caída progresiva en la cantidad y tasa de donantes, ha experimentado avances importantes en torno al significado y la responsabilidad social de la donación de órganos, dos ejemplos de ello son: La promulgación de la Ley del Donante Universal $n^{\circ} 20.413$, y la creación de la Coordinación Nacional de Procuramiento y Trasplante en el Ministerio de Salud. El primero establece claramente que toda persona mayor de dieciocho años será considerada, por el solo ministerio de la ley, donante de órganos una sin embargo la realidad es otra, ya que siempre se solicita y respeta el consentimiento expreso y firmado de la familia directa del fallecido, aun cuando obrara sin respetar la voluntad de éste, ya sea para no agravar el duelo familiar aún más o porque 
la propaganda y publicidad negativa que se pudiera derivar produciría un efecto negativo a la donación de órganos en general ${ }^{(8)}$; y el segundo otorga por primera vez una estructura ministerial y estatal a la actividad de donación-trasplante ${ }^{(9)}$.

El proceso de donación-trasplante, se inicia con la detección del donante ${ }^{(10)}$, desencadenando una serie de actividades dentro de etapas determinadas, que conllevan a la obtención del o los órganos a trasplantar.

El número de órganos disponibles está definido en base a los potenciales donantes, lo cual se entiende por aquellos usuarios con diagnóstico clínico e instrumental de muerte encefálica sin contraindicaciones para la donación ${ }^{(11)}$, y que hasta hace un tiempo correspondían a todos aquellos usuarios con injuria cerebral y Glasgow de ingreso igual o menor a 7 puntos, llamados ahora posibles donantes.

Por ello, el mantenimiento del donante se ha instaurado como una actividad dinámica y compleja, dependiente de múltiples factores, teniendo como fin asegurar la correcta perfusión y oxigenación de los órganos, que confluyen finalmente en la viabilidad de los mismos ${ }^{(12)}$, logrando así obtener el mayor número de órganos y de la mejor calidad posible, aumentando la posibilidad de éxito post trasplante ${ }^{(13)}$.

El equipo procurador y de trasplante, el cual se encuentra capacitado, motivado y cuenta con profesionales especializados $^{(14)}$, ha de estar completamente informado de la situación actual del donante, ya que una vez confirmada la muerte encefálica, se encuentra en la obligación de solicitar la donación de órganos. Difícil tarea, si se considera el estado psicológico de la familia, que viene a estar dado por una situación de shock y desesperación ${ }^{(15)}$ desencadenando en primera instancia la esencia de la negativa familiar, principal causa de pérdida de potenciales donantes.

Las razones más frecuentes que pueden ocasionar una negativa familiar, son el desconocimiento de los deseos del potencial donante con respecto a la donación(16), a las dudas acerca de la muerte del donante, por no comprender el concepto de muerte cerebral, o por miedo a una prematura declaración de muerte, mutilación o desmembramiento del cuerpo. Otras causas de negativa familiar, es el no estar mentalizados, ya que es muy corto el espacio de tiempo entre la comu- nicación de la mala noticia y la solicitud de donación, motivos religiosos porque piensan en la reencarnación y en la utilidad del cuerpo en una vida posterior, una mala información previa ${ }^{(17)}$ el miedo a la publicidad, la crítica de familiares y amigos, las diferencias de opinión entre los miembros de la familia, los roces con el personal sanitario o la creencia de que no se ha atendido correctamente al fallecido o bien un comportamiento de los profesionales sanitarios fríos, autoritario y con falta de humanidad. El nivel cultural de las familias es otro factor a considerar(18).

Finalmente, según algunos autores existen factores motivacionales que activan la conducta de donación de órganos, y los cuales se deben fomentar, tales como el conocer la experiencia de terceros, la solidaridad que despierta la persona del receptor, la familiaridad o cercanía de las personas con la donación y/o trasplante de órganos, el deseo de ayudar a quien lo necesita, y pensar que alguien cercano pudiese necesitar un trasplante ${ }^{(19)}$.

\section{Objetivos principales}

- Aplicar las etapas del proceso donación-trasplante a un caso real de un hospital en la comuna de Concepción, Chile.

- Identificar los cuidados de enfermería durante todo el proceso de donación trasplante.

\section{Descripción del caso clínico (Etapas del proceso donación trasplante):}

\section{ETAPA I:}

\section{Evaluación del posible donante de órganos}

Mujer de 46 años, casada, donante en vida, con antecedentes de epilepsia tratada hace 15 años. Presenta cefalea de 24 horas de evolución, convulsión, hemiparesia derecha y metrorragia. Ingresa en Glasgow 4, a la sala de agudos medico quirúrgico de un hospital, intubada y ventilando espontáneamente. Hemodinámicamente estable.

Al examen físico destaca piel pálida, tibia, fascie indiferente, pupilas midriáticas con anisocoria, estridor y roncos difusos, signos de decorticación y algunos de 
flexión en extremidad inferior derecha. Se le realiza una tomografía axial computarizada que revela extensa hemorragia intracerebral, e hipertensión endocraneana, sin indicación neuroquirúrgica, con pronóstico ominoso y riesgo vital inminente.

* Enfermera de procuramiento es informada vía telefónica por enfermera tratante, de la posible donante, lo cual da inicio al proceso de donación-trasplante. Esposo e hijos manifiestan el deseo del familiar de ser donante, lo cual es confirmado a través del servicio de Registro Civil e Identificación de Chile.

Intervención Enfermera de procuramiento: gestión de cama en Unidad de Cuidados Intensivos para manejo de posible donante con enfermera y médico de turno.

\section{Diagnóstico de Enfermería al ingreso:}

- Deterioro de la perfusión cerebral relacionado con hemorragia cerebral y aumento de la presión intracraneana manifestado por Glasgow 4, convulsión, pupilas midriáticas con anisocoria, piel pálida, hemiparesia derecha, signos de decorticación y flexión en extremidad inferior.

\section{Cuidados de Enfermería:}

- Valoración del estado de conciencia utilizando escala de Glasgow, signos vitales, perfusión, ventilación, movilidad y fuerza motora, sensibilidad superficial y profunda.

- Valoración frecuencia, profundidad, y ritmo de la respiración, así como también la permeabilidad y protección del tubo endotraqueal.

- Instalación de monitor cardiaco, sonda nasogastrica, sonda vesical y vía venosa.

- Realización de balance hídrico estricto.

- Administración de oxigenoterapia, medicamentos y soluciones parenterales indicados por el médico.

- Mantención de técnica aséptica, posición en ángulo de 30 a 45 grados, en cama con barandas, y ambiente tranquilo.

- Evaluación constante de la evolución, de los efectos deseados y adversos.
ETAPA II:

\section{Mantención del donante}

Posible donante ingresa a Unidad de Cuidados Intensivos, en Glasgow 4, ventilando espontáneamente, intubada, con signos de descerebración, y sin sedación. Se conecta a ventilador mecánico, por si presenta crisis de apnea, en CPAP con una presión de soporte vital igual a 8, y fracción inspirada de oxígeno al $21 \%$, saturando $99 \%$, con 19 respiraciones por minuto. Normotensa, con una presión arterial media de 100 mmhg, normocárdica y afebril. Al examen físico midriática arreactiva, yugulares planas, sin edema de extremidades. Con diuresis de $300 \mathrm{cc}$ por sonda vesical y línea arterial para monitorización continua.

Durante su evolución cursa diabetes insípida e hipernatremia de $168 \mathrm{meq} / \mathrm{l}$ (figura 1), diuresis de 4575 con balance negativo de 3107, que se maneja con desmopresina, agua libre por sonda nasogastrica y suero fisiológico al medio con 1 gramo de cloruro de potasio. Hipertensa (190/88 mmhg) con presión arterial media de 105 mmhg que tiende a disminuir, taquicardica (140 latidos por minuto) y subfebril. (37,4 grados Celsius). Destaca aumento del hematocrito (Figura 2).

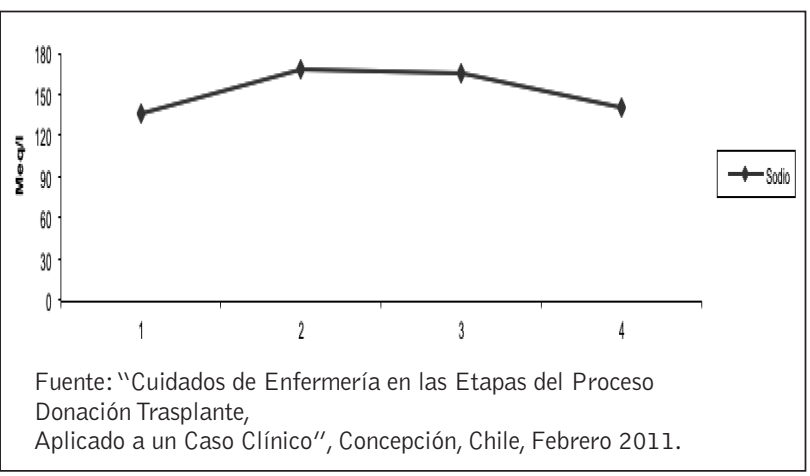

Figura 1. Curva de Sodio

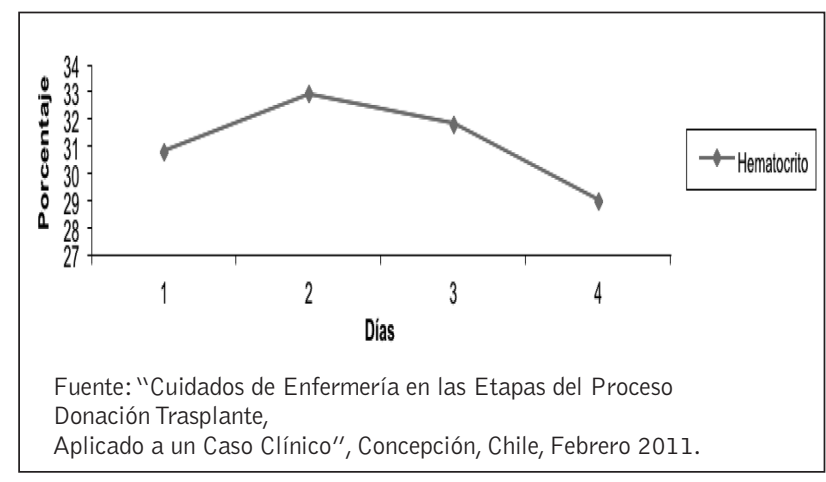

Figura 2. Curva de Hematocrito 


\section{Diagnóstico de Enfermería:}

- Déficit de volumen de líquidos relacionado a la hipernatremia y al exceso de orina secundario a la diabetes insípida manifestado por taquicardia, con tendencia a la disminución de la presión arterial media, estado subfebril, y aumento del hematocrito.

Cuidados de Enfermería: (manejo multisistémico por protocolo de UCI y manejo del edema cerebral secundario e hipertensión intracraneana)

- Monitorización continua de los signos vitales y niveles de glicemia.

- Toma de sangre para exámenes de control, valorando en particular electrolitos plasmáticos y hemograma.

- Valoración de los cambios en el estado de conciencia, pulsos periféricos, llenado capilar, hidratación de mucosas y turgencia de la piel.

- Realización balance hídrico estricto en forma horaria, considerando flujo urinario horario.

- Administración de soluciones parenterales, medicamentos y drogas vasoactivas indicados por el médico.

- Supervisión de funciones delegadas tales como cambios posturales, aseo de cavidades y genitales, baño en cama, cambios de pañales, protección de prominencias óseas y lubricación de la piel.

- Acogida a la familia y entrega de toda la información necesaria, requerida y pertinente.

- Facilidad para la visita y acompañamiento familiar.

- Evaluación constante de la evolución, de los efectos deseados y adversos.

Al examen neurológico del cuarto día posible donante se encuentra en Glasgow 3, sin respuesta motora 0 vegetativa. Con ventilación asistida, saturando $100 \%$, piel pálida, fría, con enlentecimiento del llenado capilar, hipotensa (75/47 mmhg) con una presión arterial media entre 60 y 70 mmhg, bradicardica (52 latidos por minuto) e hipotermica (33,7 grados Celsius), con una diuresis entre 70 y $50 \mathrm{cc}$ por hora. Se corrige hipernatremia, sin embargo, por inestabilidad hemodinámica test de apnea se realiza al día siguiente.

\section{Diagnóstico de Enfermería:}

- Disminución del gasto cardiaco relacionado a la alteración del volumen de eyección manifestado por piel pálida, fría, enlentecimiento del llenado capilar, disminución de los pulsos periféricos, hipotensión, hipotermia y oliguria.

\section{Cuidados de Enfermería:}

- Monitorización continua de la presión arterial, diuresis horaria, saturación de oxigeno, temperatura, niveles de hematocrito, hemoglobina y electrolitos plasmáticos.

- Control de la dosis de todas las drogas vasoactivas indicadas por el médico para mantener presión arterial igual o superior a $100 \mathrm{mmhg}$ y diuresis igual o superior a $1 \mathrm{ml} / \mathrm{kg} / \mathrm{h}$.

- Mantención de la saturación de oxigeno superior a $95 \%$, con niveles de $\mathrm{Pa02}$ igual o superior a 100 mmhg, y de PaC02 entre 35 y 45 mmhg, un $\mathrm{Ph}$ entre 7,35 y 7,45, un hematocrito superior al $30 \%$, hemoglobina superior a $10 \mathrm{~g} / \mathrm{l}, \mathrm{y}$ temperatura corporal superior a 35 grados Celsius mediante la aplicación de frazadas eléctricas.

Intervención Enfermera de procuramiento durante toda esta etapa: monitoreo continuo durante las visitas de mañana, medio día y tarde, de la hemodinamia del posible donante, de su oxigenación, del resultado de sus exámenes de control, tanto sanguíneos como neurológicos, de las indicaciones médicas y los cuidados de enfermería, todo con el fin de asegurar el funcionamiento óptimo de los órganos susceptibles a trasplantar.

\section{ETAPA III:}

\section{Diagnóstico Clínico del potencial donante}

Al quinto día, posible donante hemodinámicamente estable, sin alteraciones metabólicas ni endocrinas, sin antecedentes de ingesta de drogas depresoras del sistema nervioso central, ni de uso de bloqueadores neuromusculares, por lo que se realiza la evaluación por neurólogo para diagnostico clínico que demostró: 
1. Ausencia de respiración espontánea: a través del Test de apnea, que resultó ser positivo por una presión parcial de oxigeno mayor a $60 \mathrm{mmhg}$, y una diferencia entre la muestra basal y la 2 da muestra mayor a 20 mmhg (figura 3).

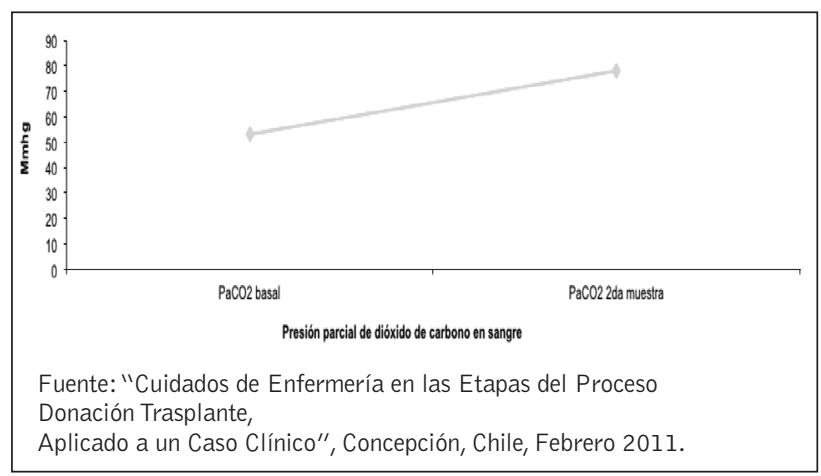

Figura 3. Curva de Presión Parcial de Dióxido de Carbono en Sangre

\section{Ausencia de funciones cerebrales}

Posible donante en coma arreactivo sin ningún tipo de respuesta motora o vegetativa al estímulo doloroso.

\section{Ausencia de reflejos troncoencefálicos} Abolición de reflejos: posible donante con midriasis fija, arreflectiva bilateral, sin reflejos corneales, oculocefálicos, y faringeos.

Conclusión diagnóstica: muerte encefálica, es decir, potencial donante.

Intervención Enfermera de procuramiento: respalda normativa vigente respecto a estabilidad hemodinámica del posible donante, equilibrio metabólico-endocrino, y ausencia de drogas depresoras para la realización del test de apnea.

\section{ETAPA IV: Consentimiento Familiar}

Previo a la realización del test de apnea al quinto día, familia solicita reunión con médico jefe de la unidad de cuidados intensivos, por el tema de donación de órganos, en la cual señalan que no aceptan la donación, pese a estar consciente de la decisión en vida de su familiar de ser donante, y de haberlo manifestado espontáneamente, lo cual se respeta dando aviso al equipo de procuramiento y trasplante, quienes suspenden el seguimiento del potencial donante. Cabe mencionar que no se dio aviso de dicha reunión a éste equipo.

Dentro de las causas de la negativa de esta familia, destaca, por un lado el reciente hecho publicado en los noticieros nacionales respecto al tráfico de órganos en un hospital de la capital, puesto dejo un precedente importante ya que la familia en todo momento insistía en que no le fueran a extraer nada a su familiar sin su autorización, e inclusive cada vez que entraban en horario de visita examinaban a su familiar; y por otro lado la influencia de una de las hermanas de la potencial donante en la decisión del cónyuge e hijos.

Por tanto, el proceso donación-trasplante, se detiene en esta etapa, surgiendo algunas interrogantes que quedan abiertas al debate: aunque la familia hubiese solicitado la reunión con el médico jefe de la unidad, ¿Es el más indicado para conversar con ésta acerca de la decisión que han tomado?, ¿Habrá sido mejor, en base a la experiencia, coordinarse con el equipo de procuramiento y trasplante para que hubiese estado presente durante dicha reunión? Respecto a las leyes actuales en Chile ¿Por qué se acepta la decisión tomada por la familia si el familiar fallecido es donante? ¿Por qué los profesionales sanitarios involucrados no se ampararon bajo lo que establece la ley? ¿Por qué el procurar por otras vidas no pasó a primer plano?.

\section{Conclusiones:}

- Con el transcurso de los años, si bien Chile ha avanzado en el desarrollo de nuevas políticas y lineamientos ministeriales en el tema de la donación de órganos, sobre todo en lo que respecta al proceso, no se pueden aplicar en su totalidad, ya que cultural y socialmente la familia es quien toma la última decisión, haya sido donante o no el familiar, lo que conlleva a que las listas de espera para trasplantes sigan creciendo y los usuarios con enfermedad renal crónica terminal sigan expectantes.

- Para que el proceso donación trasplante, que comienza con la pesquisa del posible donante, sea exitoso, es importante trabajar como equipo multidisciplinario en cada una de las diferentes etapas, puesto requieren de un monitoreo adecuado de lo cual dependerá la viabilidad y sobrevida del órgano a trasplantar. Dentro de este mismo proceso, mantener informada 
a la familia en todo momento, resulta vital dada su importancia en la decisión final, debiéndose contar, por tanto, con un personal sanitario capacitado, que sea capaz de comunicarse de manera asertiva y eficaz.

- Finalmente, si se actúa como equipo sanitario de manera comprometida, humana, responsable, con principios éticos-morales, y sobre todo como comunicadores sociales, se podrá crear conciencia y cultura de la donación de órganos, logrando aumentar las tasas de donación, la esperanza de vida de aquellas personas que dependen de un trasplante y mejorar la calidad de vida de aquellos usuarios que se encuentran en otras alternativas de sustitución renal.

Recibido: 5 Octubre 2011

Revisado: 10 Diciembre 2011

Modificado: 14 Agosto 2012

Aceptado: 30 Agosto 2012
5. Flores A, Lozano G, Vásquez M, Sifuentes B, Espinosa E. Actitud de la Gente ante la Donación de Órganos y Tejidos. México: Universidad autónoma de San Luis de Potosí; Universidad autónoma de Zacatecas; 2008.

6. Manyalich M. Evolución del procuramiento de órganos en el mundo. Rev. Médica Clínica las Condes (revista en internet)* 2010. (acceso 01 de marzo 2011); 21(2). Disponible en: http:// www.clinicalascondes.com/area-academica/pdf/ MED_21_2/1_Evoluciondel_Manyalich.pdf.

7. Modificación de la ley $n^{\circ} 19.451$, con el fin de determinar quiénes pueden ser considerados donantes de órganos y la forma en que pueden manifestar su voluntad. Ley 20.413/2010 de 15 de enero. Diario Oficial de Chile, (16-012010).

8. López A, Kulisevsky J, Caballero F. El donante de órganos y tejidos. Evaluación y manejo. Barcelona: Springer-Verlag Ibérica; 1997.

9. Servicio de Salud Concepción. Orientaciones Técnicas y Compromisos de gestión 2011. Chile: Oficina de Coordinación Nacional de Procuramiento y Trasplante de Órganos y Tejidos; 2011. Versión preliminar.

10. Martínez P. Detección de donantes. En: Tercer Congreso Internacional de Medicina Crítica. España; Universidad de Cádiz; 2002.

11. Ministerio de Salud. Programa de Calidad de generación de donantes. Chile: Coordinación Nacional de Trasplantes; 2011.

12. Uribe M. Guías Clínicas sobre Procuramiento de Órganos y Trasplante. Chile: Sociedad Chilena de Trasplante; 2010.

13. Rojas J. Selección y manejo del potencial donante de órganos. Rev Hospital Clínico Universidad de Chile (revista en internet)* 2007. (acceso el 01 de marzo de 2011); 18. Disponible en: http://www.redclinica.cl/HospitalClinicoWebNeo/Controls/Neochannels/Neo_CH6258/deploy/selec_manejo_potenc.pdf. 
14. Servicio de Salud Concepción. Manual de Procuramiento de Órganos y Tejidos para Trasplante. Versión actualizada. Chile: Corporación del Trasplante; 2010.

15. Leal E, Komatsu M-C. El rechazo familiar al pedido de donación de órganos y tejidos para transplante. Rev Latino-am Enfermagem (revista en internet)* 2008 mayo-junio. (acceso 01 de marzo de 2011); 16(3). Disponible en: http://www. scielo.br/pdf/rlae/v16n3/es_20.pdf.

16. González K, Silva C, Catoni M, Y Palma E. Causas de Negativa Familiar y Estrategias para Aumentar la Donación De Órganos, en Familiares de Donantes Cadavéricos. Revista de la Sociedad Chilena de Enfermería en Diálisis y Trasplante Renal (revista en internet)* 2005. (acceso 01 de marzo de 2011); 3(6). Disponible en: http:// senferdialt.cl/pdf/jornadas/Numero6-2005.pdf.
17. Lozano G. Actitudes y Persuasión hacia la Donación de Órganos y Tejidos. (resumen en Internet)*. México: Facultad de Psicología, Universidad Nacional Autónoma; 2003 (acceso 24 de marzo de 2011) Disponible en: http://www.uaq. $\mathrm{mx} /$ psicologia/lamision/donacion_organos.html.

18. Luque $E$, Matamala $A$, Torres $A$, Barroso $R$, Sánchez $\mathrm{Y}$, Ferret $A$, et al. Evaluación de un programa educativo sobre la donación de órganos dirigido a escolares. Revista de la Sociedad Española de Enfermería Nefrológica (revista en internet)* 2005. (acceso 01 de marzo de 2011); 9(3). Disponible en: http://scielo.isciii.es/pdf/nefro/v9n3/original4.pdf.

19. Bustamante M, Villarreal P. Motivación a la donación de órganos en Chile. Panorama socioeconómico año 26 (revista en internet)* 2008 enero-junio. (acceso 24 de marzo de 2011); 26(36). Disponible en: http://redalyc.uaemex.mx/pdf/399/39903608.pdf. 